\title{
Haze Removal and Color Compensation of Underwater Image
}

\author{
Nisha Kumari \\ Department of Electronics and Communication \\ Engineering \\ Dr. B. R. Ambedkar National Institute of Technology \\ Jalandhar, (Punjab) - 144011
}

\author{
Ramesh Kumar Sunkaria \\ Department of Electronics and Communication \\ Engineering \\ Dr. B. R. Ambedkar National Institute of Technology \\ Jalandhar, (Punjab) - 144011
}

\begin{abstract}
In underwater image capturing, color change and presence of haze are the two sources of distortion. These distortions are caused by light scattering and light attenuation occurred by light traveling in water with different wavelengths. These changes are caused by light incident on objects, reflected and deflected by different particles present in the propagation path before reaching the camera. Thus the images are hazy and have bluish tone. In the present work, haze removing technique proposed to enhance the image and to compensate the other colors which have disappeared. In the present work, the distortion caused by artificial light, haze effect and appearance of bluish tone are compensated. Based on the amount of attenuation corresponding to each wavelength, color change compensation is conducted and color balance is restored. Effect of noise is also reduced by using the spatial filter. Using this technique the visibility and color of the image can be enhanced.
\end{abstract}

\section{Keywords}

Color compensation, denoising, light attenuation, light scattering, haze.

\section{INTRODUCTION}

Underwater image capturing is very important for the scientist. Autonomous Underwater Vehicles and Remotely Operated Vehicles are used for taking the images such as underwater mines, shipwrecks, coral reefs, pipelines, ocean engineering, monitoring sea life in the sense of geological and biological interpretation and navigation purpose [1], [2], [3]. In underwater environment various hazy images occur, caused by light that is reflected from a surface and is scattered by water particles. Underwater images are dominated by blue color, because of varying degrees of attenuation for different wavelengths i.e. red, green and blue [4]-[6]. This lowers the visibility and contrast.

Because of sand, minerals, plankton in ocean, river and lakes haze occurs. During image capturing, light is reflected from the object propagates towards the camera and in propagation path these particles absorb and scatter the light beam. In the absence of blackbody radiation [7], the multi-scattering process along the course of propagation further disappear the beam into homogenous background light. There are several techniques targeted on the removal of light scattering - [8],
[9], [10]. Here visibility increases and contrast enhancement occurs but distortion caused by attenuation, i.e. color change remains present. [11], [12] and [13] are used for compensation of color but blurriness occurs caused by light scattering. Images collected by image sensors are generally affected by noise and noise in image can be introduced by transmission errors and compression. The problem of image denoising is to recover an image that is cleaner than its noisy observation. Thus, noise reduction is an important technology in underwater image analysis and the first step to be taken before images are analyzed [20]. The proposed algorithm is used to remove distortion caused by light scattering and light attenuation. By using this algorithm, there are no requirements of expensive optical instruments or stereo image pairs.

\section{UNDERWATER MODEL}

Underwater image formation model is shown in figure 1. Here natural light enters from air to an underwater scene point $\mathrm{x}$ and light reflected from the object and propagates distance $\mathrm{d}(\mathrm{x})$ to the camera. The light reached to the camera is the sum of the background light formed by multi-scattering and the direct transmission of reflected light.

Since water is denser than air, so that when light propagates from air to water, it's some part is reflected back and some part entered the water [12]. The amount of light that enters the water also starts attenuated as going towards more depth in the sea [9]. The water molecules and particles inside the water also absorb certain amount of light [12]. There is also disappear colors one by one depending on the wavelength of the color as shown in figure 2. The blue color travels the longest in water due to its shortest wavelength. So that underwater images are dominated by blue color. Result, blur images contain low brightness, low contrast and less visibility.

Hazy image formation model gives the simplified equation [15], [16]:

$$
I_{\lambda}(x)=J_{\lambda}(x) \cdot t_{\lambda}(x)+\left(1-t_{\lambda}(x)\right) \cdot A_{\lambda}, \lambda \in\{R, G, B\}
$$




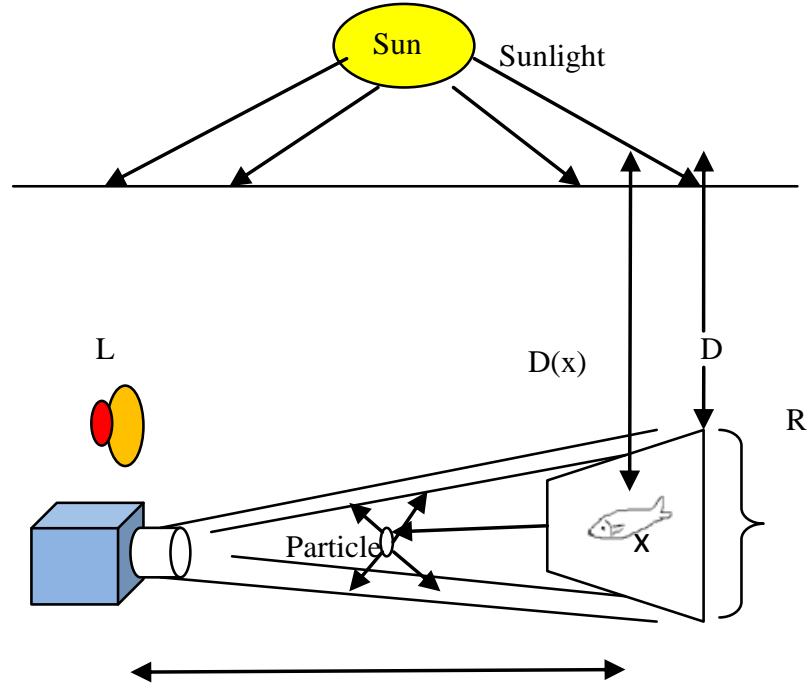

Object camera distance $\mathrm{d}(\mathrm{x})$

Figure 1 Underwater image formation model

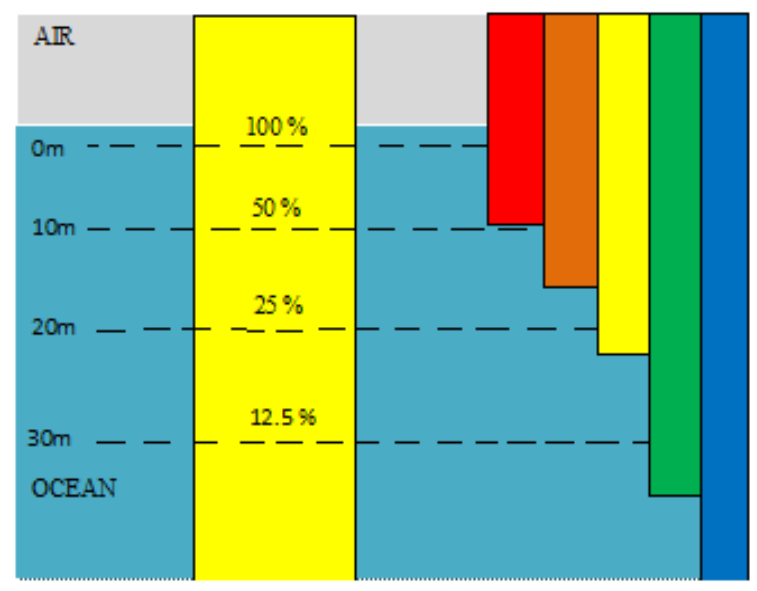

Figure 2 Attenuation for different wavelength in water

where $\mathrm{x}=$ point in the underwater scene, $\mathrm{I}_{\lambda}(\mathrm{x})=$ image captured by the camera, $\mathrm{J}_{\lambda}(\mathrm{x})=$ scene radiance, $\mathrm{t}_{\lambda}=$ residual energy ratio of $\mathrm{J}_{\lambda}(\mathrm{x})$ after reflecting from point $\mathrm{x}$ in the underwater scene and reaching the camera, $A_{\lambda}=$ homogenous background light, $\lambda=$ wavelength of light, $\mathrm{J}_{\lambda}(\mathrm{x}) \cdot \mathrm{t}_{\lambda}(\mathrm{x})=$ decay of scene radiance in the water [17].

$t_{\lambda}(x)=\frac{E_{\lambda}^{\text {residual }}(x)}{E_{\lambda}^{\text {initial }}(x)}=10^{-\beta(x) \cdot d(x)}=\operatorname{Nrer}(\lambda)^{d(x)}$ where $\operatorname{Nrer}(\lambda)=$ normalized residual energy $=$ ratio of residual to initial energy for every unit of distance propagated, $\beta(\lambda)=$ medium extinction coefficient [16]. This is affected by wavelength, salinity and concentration of phytoplankton [18]. For every meter most clear ocean water the value of $\operatorname{Nrer}(\lambda)$ is 0.8 to 0.85 when $\lambda$ is $650 \mu \mathrm{m}$ to $750 \mu \mathrm{m}, 0.93$ to 0.97 when $\lambda$ is $490 \mu \mathrm{m}$ to $550 \mu \mathrm{m}$ and 0.95 to .99 when $\lambda$ is $400 \mu \mathrm{m}$ to $490 \mu \mathrm{m}$.

\section{METHODOLOGY}

The proposed algorithm is shown in flow diagram figure 3 . Here, suppose the artificial light $\mathrm{L}$ is present and aim is to remove the light scattering and color change that occurred along the propagation path $\mathrm{d}(\mathrm{x})$ from the object to the camera and after that compensation of the distortion caused by wavelength attenuation for traversing the water depth ' $D$ ' i.e. surface of the water to the top of the image. Now equation (1) can be written as energy model as follows:

$I_{\lambda}(x)=\left(E_{\lambda}^{A}(x) \cdot \operatorname{Nrer}(\lambda)^{D(x)} \cdot \rho_{\lambda}(x)\right) \cdot \operatorname{Nrer}(\lambda)^{d(x)}+\left(1-\operatorname{Nrer}(\lambda)^{d(x)}\right) \cdot A_{\lambda}, \lambda \in\{R, G, B\}$

Here the color change occurs not only along the object camera route but also along the surface object propagation path. Once the scene depth, i.e. object camera distance $\mathrm{d}(\mathrm{x})$ is known through the "dark channel prior". The value $\operatorname{Nrer}(\lambda)^{\mathrm{d}(\mathrm{x})}$ can be calculated. Thus the direct attenuation term $E_{\lambda}^{A} \cdot \operatorname{Nrer}(\lambda)^{D(x)} \cdot \rho_{\lambda}(x)$ is derivable through dehazing procedure. The surface object distance ' $\mathrm{D}(\mathrm{x})$ ' is calculated by comparing the residual energy ratio of different color channels. For a given water depth $\mathrm{D}(\mathrm{x})$ the amount of reflecting light $E_{\lambda}^{A}(x) \cdot \rho_{\lambda}(x)$ i.e. free of light scattering and color change, from point $\mathrm{x}$ illuminated by airlight, is determined [19].

The artificial light source $\mathrm{L}$ is provided to compensate insufficient lighting under the water. The luminance contributed by the artificial light source has to be removed before the dehazing and color compensation. When considering artificial light the equation becomes:

$I_{\lambda}(x)=\left(\left(E_{\lambda}^{A}(x) \cdot \operatorname{Nrer}(\lambda)^{D(x)}+E_{\lambda}^{L} \cdot \operatorname{Nrer}(\lambda)^{d(x)}\right) \cdot \rho_{\lambda}(x)\right) \cdot \operatorname{Nrer}(\lambda)^{d(x)}+\left(1-\operatorname{Nrer}(\lambda)^{d(x)}\right) \cdot A_{\lambda}, \lambda \in\{R, G, B\}$

Above model shows the hazing effect, wavelength attenuation and artificial light effect. Now the given steps used to calculate the distance $\mathrm{d}(\mathrm{x}), \mathrm{D}(\mathrm{x})$, presence of artificial light, depth range ' $R$ ' and the corresponding procedure for dehazing color compensation and denoising. 


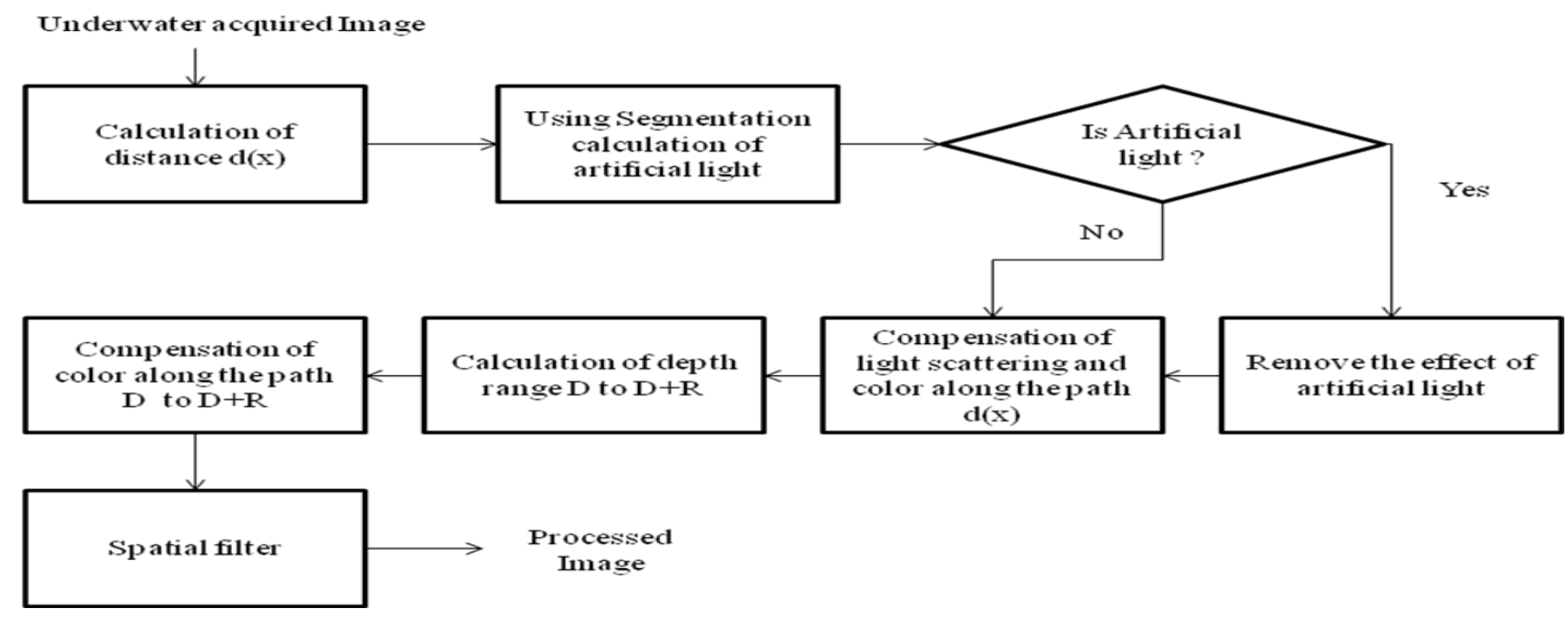

Figure 3 Block diagram of propossed algorithm

\subsection{Calculation of Distance between Object and Camera Using Dark Channel Prior Method:}

There is a relation between haze and distance, i.e. more distant objects are more hazier [14]. So, evaluating the amount of haze in a single image is sufficient to find the distance $d(x)$ [22]. For finding distance $d(x)$, dark channel prior method [9] is used. In most of the non-background light patches $\Omega(\mathrm{x})$, where $\mathrm{x} \in \Omega(\mathrm{x})$, on a haze free underwater image, at least one color channel has a very low intensity at some pixels. If no pixels with a very low intensity value can be found in the local patch, which implies the existence of haze. So considering, point $\mathrm{x}$ is a part of foreground object then

$J_{\text {dark }}(x)=\min _{\lambda} \min _{y \in \Omega(x)} J_{\lambda}(y), \lambda \in\{R, G, B\}$.

$\min _{y \in \Omega(x)}\left(I_{\lambda}(y)\right)=\min _{y \in \Omega(x)}\left\{J_{\lambda}(y) \cdot \operatorname{Nrer}(\lambda)^{d(y)}+\left(1-\operatorname{Nrer}(\lambda)^{d(y)}\right) \cdot A_{\lambda}\right\}, \lambda \in\{R, G, B\}$.

$\min _{\lambda}\left(\operatorname{Nrer}(\lambda)^{d(x)}\right)=1-\min _{\lambda}\left\{\frac{\min _{y \in \Omega(x)}\left(I_{\lambda}(x)\right)}{A_{\lambda}}\right\}, \lambda \in\{R, G, B\}$

where,

$A_{\lambda}=\max _{x \in I} \min _{y \in \Omega(x)} I_{\lambda}(y), \lambda \in\{R, G, B\}$.

Here there are put the all known values and get the value of $\mathrm{d}(\mathrm{x})$ [9].

If an artificial light source L is employed during the image capturing process, the effect of artificial light must be deducted first to avoid over-compensation. Its existence can be determined by comparing the difference between mean luminance of foreground and background. Once presence of artificial light source is detected, then it has to be removed. Reflectivity of object can be calculated at all pixels for every wavelength i.e. R, G and B [19] is as shown below:
$\rho_{\lambda}(x)=\frac{\left(I_{\lambda}(x)-\left(1-\operatorname{Nrer}(\lambda)^{d(x)}\right)\right) \cdot \frac{A_{\lambda}}{\operatorname{Nrer}(\lambda)^{d(x)}}}{E_{\lambda}^{A}(x) \cdot \operatorname{Nrer}(\lambda)^{D(x)}+E_{\lambda}^{L} \cdot \operatorname{Nrer}(\lambda)^{d(x)}}, \lambda \in\{R, G, B\}$

Now,

$\dot{I}_{\lambda}(x)=I_{\lambda}(x)-\left(\left(E_{\lambda}^{L} \cdot \operatorname{Nrer}(\lambda)^{d(x)}\right) \cdot \rho_{\lambda}(x)\right) \cdot \operatorname{Nrer}(\lambda)^{d(x)}$

\subsection{Compensation of Light Scattering along the Path Object and Camera:}

Now compensation of light scattering term and color compensation as

$\bar{I}_{\lambda}(x)=I_{\lambda}(x)-\left(1-\operatorname{Nrer}(\lambda)^{d(x)}\right) \cdot A_{\lambda}=\left(\left(E_{\lambda}^{A}(x) \cdot \operatorname{Nrer}(\lambda)^{D(x)}\right) \cdot \rho_{\lambda}(x)\right) \cdot \operatorname{Nrer}(\lambda)^{d(x)}$

Thus,

$\bar{J}_{\lambda}(x)=E_{\lambda}^{A}(x) \cdot \operatorname{Nrer}(\lambda)^{D(x)} \cdot \rho_{\lambda}(x)=\frac{\bar{I}_{\lambda}(x)}{\operatorname{Nrer}(\lambda)^{d(x)}}, \lambda \in\{R, G, B\}$.

This result gives the image after compensation of light scattering and bluish tone along the path, object camera distance. Here aim is to calculate the value of $E_{\lambda}^{A}(x) \cdot \rho_{\lambda}(x)$.

\subsection{Compensation of Light attenuation along the Path Surface to Object and Denoising of the Enhanced Image}

Now there is need of compensation of the attenuation caused by $\mathrm{D}(\mathrm{x})$. Let the energy corresponding to $\mathrm{R}, \mathrm{G}$, and $\mathrm{B}$ channels above the water surface is $E_{R}^{A}, E_{G}^{A}, E_{B}^{A}$. Its value depends on the weather condition above the water surface. After penetration with depth $\mathrm{D}$, the energy of each color channel attenuated to $E_{\lambda}^{w}, \lambda \in\{\mathrm{R}, \mathrm{G}, \mathrm{B}\}$. So D is calculated as using given equation 
$\min _{D} \sum\left\|E_{\lambda}^{w}-E_{\lambda}^{A} \cdot \operatorname{Nrer}(\lambda)^{k}\right\|^{2}=0, \lambda \in\{R, G, B\}$.

After the calculation of D then,

$\hat{J}_{\lambda}(x)=\frac{\bar{J}_{\lambda}(x)}{\operatorname{Nrer}(\lambda)^{D}}, \lambda \in\{R, G, B\}$

This is the result after haze removal and calibration of color change. However, the water depths at top and bottom of the scene are not same always, so that it has to be compensate the color change at entire range $\mathrm{R}$ of image. So there is to calculate depth for each point in the image is necessary. Using linear interpolation method $\mathrm{D}(\mathrm{x})$ can be calculated:

$$
D(x)=D+R \cdot \frac{\left(a_{x}-b\right)}{(c-b)}, b \leq a_{x} \leq c
$$

where $\mathrm{D}=$ incident light traverses from the surface of water reaching the image, $\mathrm{R}=$ range of water depth covered in the image, pixel $\mathrm{x}$, top and bottom background pixels are located on scan line $\mathrm{a}_{\mathrm{x}} \mathrm{b}$ and $\mathrm{c}$ respectively.

Once $\mathrm{D}(\mathrm{x})$ is derived then it gives the exact value as using the given equation:

$$
\hat{J}_{\lambda}(x)=\frac{\hat{J}_{\lambda}(x)}{\operatorname{Nrer}(\lambda)^{D(x)}}=E_{\lambda}^{A}(x) \cdot \rho_{\lambda}(x), \lambda \in\{R, G, B\} .
$$

Here the basic approach of image denoising method is applied. So there is the spatial filter for image denoising is used. When image is passed through the filter, it gives the clearer underwater image. By using this method the effect of noise can be decreases and increase the signal to noise ratio and give the better quality with respect to other method.

\section{EXPERIMENTAL RESULT AND DISCUSSION}

The performance of the proposed algorithm is evaluated both objectively and subjectively. Both results show the superior haze removing and color balancing capability. This algorithm enhances the visibility, bluish tone, and contrast of the image as shown in figure 4 , figure 5 , and figure 6 . By using this algorithm the signal to noise ratio of the image can be increases. Table 1 , shows the variation of the signal to noise ratio. Here the improvement of signal to noise ratio can be seen in the figures which is given below i.e. figure 4 , figure 5 and figure6. Figure 4, figure 5 and figure 6 show the comparison between the dark channel prior method and the proposed algorithm. When dark channel prior method is used, it increases the contrast of the image but bluish tone remains present. Here by using the proposed algorithm, processed image is clear. Figure 4 show the effect of artificial light is balanced and clear. Here the effect of haze, Bluish tone affect and the effect of noise, which is produced in image acquiring, are decrease more. These images look like as it is not an underwater image. (a). Urigınal ımage

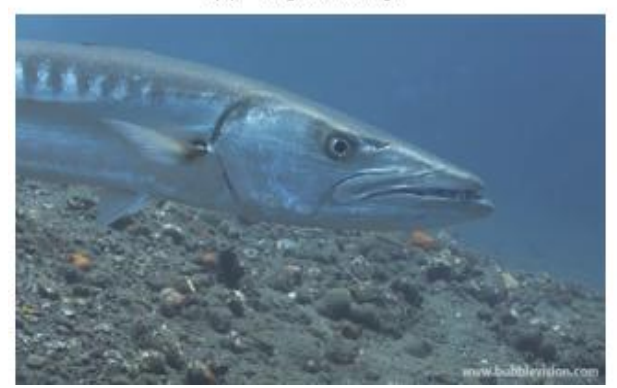

(b). Image after haze removal

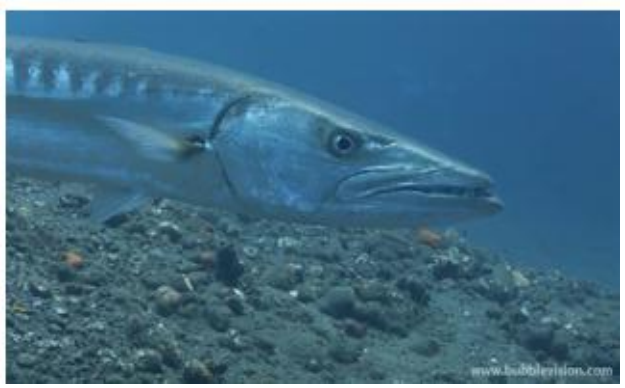

(c)lmage after proposed algorithm

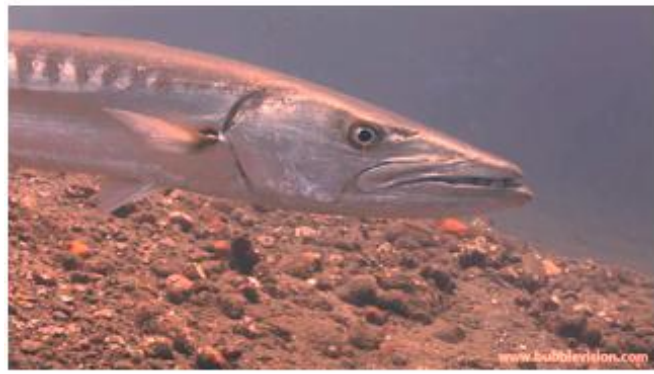

Figure 4 Image1

Table 1 Comparison of SNR between Original Image, image obtained by dark channel prior method and Image obtained by proposed algorithm

\begin{tabular}{|l|l|l|l|}
\hline S. No. & $\begin{array}{l}\text { Original } \\
\text { Image (dB) }\end{array}$ & $\begin{array}{l}\text { Image } \\
\text { Obtained } \\
\text { by Dark } \\
\text { channel } \\
\text { prior }\end{array}$ & $\begin{array}{l}\text { Image obtained } \\
\text { by Processed } \\
\text { algorithm (dB) }\end{array}$ \\
\hline 1.Image1 & 9.7882 & 10.4855 & 12.5848 \\
\hline 2.Image2 & 10.1695 & 9.6298 & 12.1314 \\
\hline & & & \\
3.Image3 & 10.5563 & 9.5253 & 11.3146 \\
\hline
\end{tabular}


(a). Original image

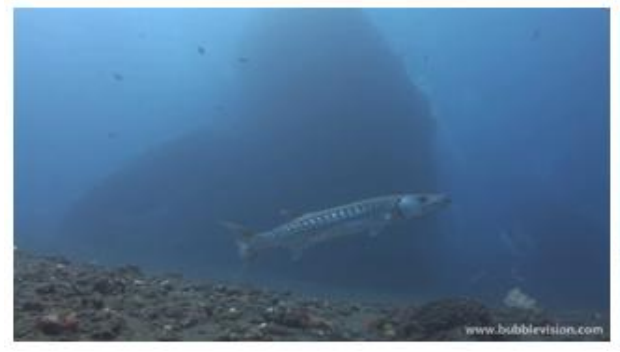

(b). Image after haze removal

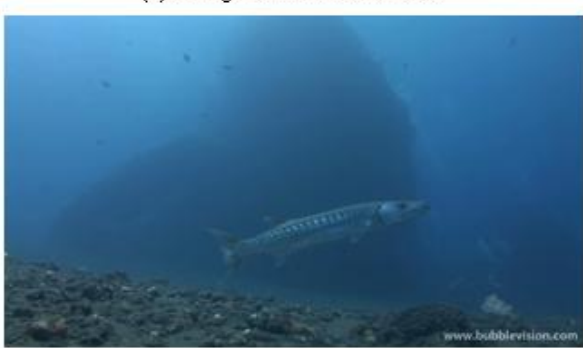

(c). Image after proposed algorithm

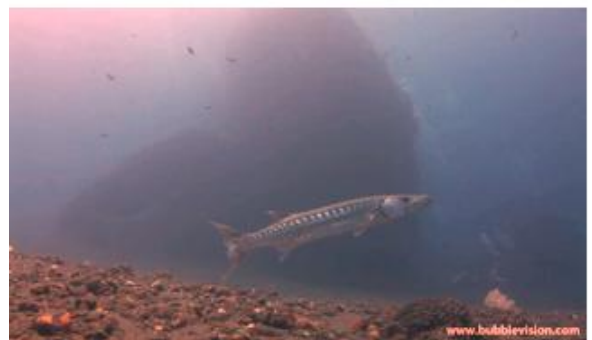

Figure 5 Image 2

(a). Original image

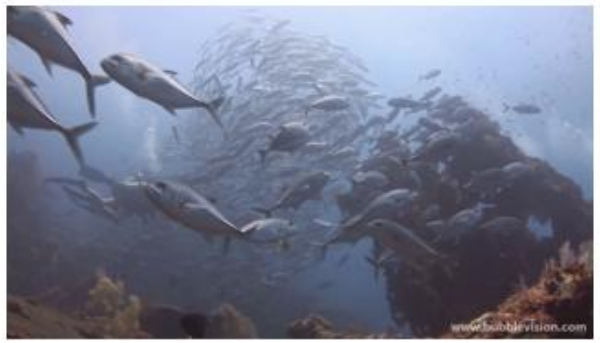

(b). Image after haze removal

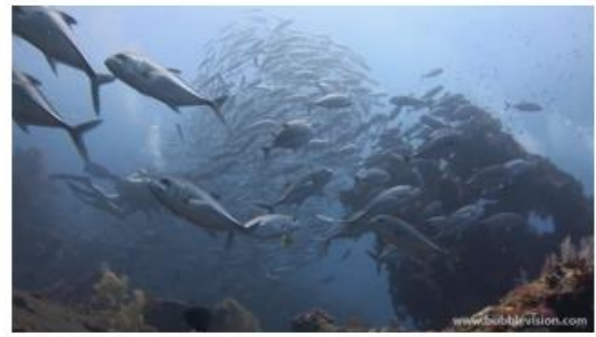

(c). Image after proposed algorithm

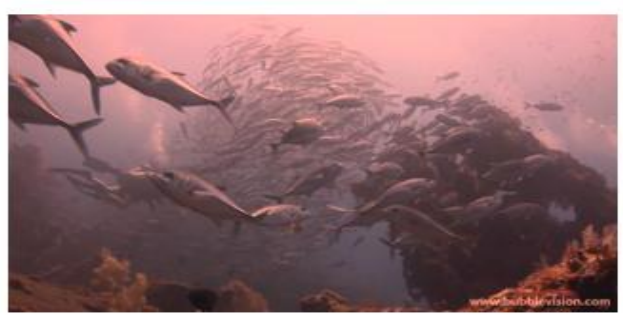

Figure 6 Image 3 


\section{CONCLUSION}

The proposed algorithm is effectively suitable for restoration of better quality of underwater image. By using this algorithm, haze, bluish tone and the effect of noise can be reduced. The experimental result shows the clarity, visibility and contrast of the underwater image. The value of extinction coefficient is different for different types of water (lake water, river water, ocean water), because the salinity of water is different. The value of extinction coefficient can vary according to water type. The processed image can vary according to artificial light change and the value of the atmospheric light i.e. weather is cloudy or day or night. This algorithm gives improved underwater image.

\section{FUTURE WORK}

It can be applied for video processing in future. Underwater image can be enhanced further to be haze free, color compensated and denoised image. Here the wavelet theory can also be applied for reducing the effect of noise in underwater images.

\section{ACKNOWLEDGMENTS}

The authors are thankful to Department of Electronics \& Communication Engineering and administration of Dr. B R Ambedkar National Institute of Technology, Jalandhar (Punjab) for providing every kind of technical and administrative support for the present work carried out in its Image Processing.

\section{REFERENCES}

[1] Prabhakar C. J., and Praveen kumar P. U., "An image based technique for enhancement of underwater images" IJMI vol. 3, Issue 4, 2011, pp-217-224.

[2] K. Lebart, C. Smith, E. Trucco, and D. M. Lane, "Automatic indexing of underwater survey video: algorithm and benchmarking method," IEEE J. Ocean. Eng., vol. 28, no. 4, pp. 673-686, Oct. 2003.

[3] J. Yuh and M. West, "Underwater robotics," Adv. Robot., vol. 15, no. 5, pp. 609-639, 2001.

[4] J. R. Zaneveld and W. Pegau, "Robust underwater visibility parameter," Opt. Exp., vol. 11, no. 23, pp. 2997-3009, 2003.

[5] E. Trucco and A. T. Olmos-Antillon, "Self-tuning underwater image restoration," IEEE J. Ocean. Eng., vol. 31, no. 2, pp. 511-519, Apr.2006.

[6] J. S. Jaffe, "Computer modeling and the design of optimal underwater imaging systems," IEEE J. Ocean. Eng., vol. 15, no. 2, pp. 101-111, Apr. 1990.

[7] M. C.W. van Rossum and T. M. Nieuwenhuizen, "Multiple scattering of classical waves: Microscopy, mesoscopy and diffusion," Rev.Modern Phys., vol. 71, no. 1, pp. 313-371, Jan. 1999.
[8] Y. Y. Schechner and N. Karpel, "Recovery of underwater visibility and structure by polarization analysis," IEEE J. Ocean. Eng., vol. 30, no. 3, pp. 570587, Jul. 2005.

[9] L. Chao and M. Wang, "Removal of water scattering," in Proc. Int. Conf. Comput. Eng. Technol., 2010, vol. 2, pp. 35-39.

[10] W.Hou,D. J.Gray,A.D.Weidemann,G. R. Fournier, and J. L. Forand,"Automated underwater image restoration and retrieval of related opticalproperties," in Proc. IGARSS, 2007, vol. 1, pp. 1889-1892.

[11] A. Yamashita, M. Fujii, and T. Kaneko, "Color registration of underwaterimage for underwater sensing with consideration of light attenuation,"in Proc. Int. Conf. Robot. Autom., 2007, pp. 4570-4575.K.

[12] Iqbal, R. Abdul Salam, A. Osman, and A. Zawawi Talib, "Underwaterimage enhancement using an integrated color model," Int. J. Comput. Sci., vol. 34, no. 2, pp. 2 12, 2007.

[13] I. Vasilescu, C. Detwiler, and D. Rus, "Color-accurate underwater imaging using perceptual adaptive illumination," in Proc. Robot. Sci.Syst., Zaragoza, Spain, 2010.

[14] K. He, J. Sun, and X. Tang, "Single image haze removal using DarkChannel Prior," in Proc. IEEE CVPR, 2009 vol. 1, pp. 1956-1963.

[15] S. Shwartz, E. Namer, and Y. Y. Schechner, "Blind haze separation," in Proc. IEEE CVPR, 2006, vol. 2, pp. 1984-1991

[16] .J. T. Houghton, The Physics of Atmospheres, 2nd ed. Cambridge, U.K.: Cambridge Univ. Press, 2001, ch. 2.

[17] W. N. McFarland, "Light in the sea-Correlations with behaviorsof fishes and invertebrates," Amer. Sci Zoology, vol. 26, no. 2, pp. 389-401, 1986.

[18] S. Q. Duntley, "Light in the sea," J. Opt. Soc. Amer., vol 53, no. 2, pp. 214-233, 1963.

[19] John Y. Chiang and Ying-Ching Chen, "Underwater image enhancement by wavelength compensation and image dehazing" in Proc. IEEE J. on IP, vol.21, no. 4 april 2012.

[20] Y. H. Lee and S. B. Rhee, "Wavelet-based image denoising with optimal filter" on International Journal of information processing system, vol. 1,2005.

[21] [Online]. Available: http://www.youtube.com/user/bubblevision.

[22] R. Fattal, "Single image dehazing," in Proc. Int. Conf. Comput. Graph. Interact. Tech., 2008, pp. 1-9. 